\title{
Encoding of Multifaceted Images using Pattern Matching Techniques
}

\author{
K. Uma*, B.Vignesh and L. Ranjith Reynold \\ Department of IT, VIT University,Vellore - 632014, Tamil Nadu, India; drumakphd@gmail.com \\ vigneshbvpro@gmail.com, ranjithreynold75@gmail.com
}

\begin{abstract}
The Communication of Scanned document needs high bandwidth due to the high volume of data and large size of the document. In order to optimize the bandwidth in uploading, downloading and exchanging the operation in multimedia communication, compression techniques are essential. Many compression techniques are already available in the literature such as Multi-dimensional Multi-scale Parser (MMP), JPEG-2000, Digipaper, DjVu, and H.264/AVC. However the compression ratio and PSNR values provided by them are not sufficient to handle the large volume of documents. Hence a new compression algorithm called enhanced-MMP is proposed in this paper by enhancing the existing MMP, R-D segmentation and optimization techniques have been used in this work and the compression algorithm uses quad tree based segmentation for smooth image and binary tree based segmentation for non-smooth image. This greatly reduces the complexity of segmentation and finally an adaptive dictionary matching technique is used for matching the pattern effectively, which contains different scale dimensions. This algorithm provides better performance than the existing algorithms with respect to compression ratio and PSNR.
\end{abstract}

Keywords: Dictionary Revision Method, Pattern Matching and EMMP, Predictive Coding, Quad Tree Segmentation, R-D Segmentation, R-D Optimization

\section{Introduction}

System generated images are assorted with text, graphics, and natural pictures leading to high volume of data. An added category of compound images are scanned document images, where compression techniques are intensively applied. In order to relate different compression algorithms to different image types, habitually a scanned image is first segmented into different module earlier than compression. Layer-based and block-based techniques are two most important methods are recurrently used. The majority layer-based approaches use the typical 3-layer Mixed Raster Content (MRC) segmented into the background and foreground, and the mask ${ }^{1}$. This segmentation is very complicated method, which involves associating mechanism, outline cohesiveness, and symbol comparison, the second approach is Block-based segmentation which is used for scanned images, and this method gives the less complexity and a more spatial resolution. To prevail over this drawback, we apply rate-distortion optimized segmentation by using block thresholding and to categorize each $16 \times 16$ block into single of the four categories: smooth (picture), text, graphics (multiple color), and image block. Moreover, the compression techniques must not only provide high compression ratios, but also encompass low complexity and visually lossless quality. Reduction in complexity is extremely significant for real-time application. In such scenario, sharp image quality reduces the readability of the text. In general, Scanned images are captured through an electronic imaging process, while the processor displays images that are basically artificial images. The existing Photographic image compression techniques, such as or JPEG-2000, JPEG (Join Photography expert group) are still pertinent to scanned images, moreover, their presentation is improved via adopting dissimilar individuality for text/graphics and pictures 2 . 
Most of the scanned compound documents use the image compression techniques like JPEG Algorithm. In this paper, input images are divided into background and foreground layers by the use of morphological operator that uses arithmetic encoder for mask layers. The Scanned images typically encompass privileged spatial resolution. The least amount for adequate quality of scanned images is $300 \mathrm{dpi}$ (dots per inch), the other normal images, it is less than $100 \mathrm{dpi}^{3}$. Block-based approaches are suitable for handled scanned images. The other criteria for scanned images consistently include a few amount of noise. The existing compression algorithm called Multidimensional Multi scale Parser (MMP) algorithm gives the results with high complexity and average compression ratio, In order to overcome such drawback; we propose a low complexity and a high quality compression encoder in this paper which is called Enhanced Multidimensional Multiscale Parser (EMMP). In this algorithm, block segmentation method is used to segment the original image into text/ graphics, pictures and complex blocks. Rate Distortion Optimization (RDO) classification algorithm is used in this work for further classification of complex blocks. Then the complex blocks are divided into complex text and complex picture blocks based on $\mathrm{RDO}$ techniques. Finally concatenation of text blocks and image blocks is performed. This EMMP compression technique has been applied to both text and images in the blocks carried out in this work. Moreover, this method is used to compress the variations of multimedia images. The EMMP includes tree based segmentation with multiscale approximate block matching attached with an adaptive dictionary. Mainly, we focus in this paper is to reduce the complexity and to increase the quality of images ${ }^{4}$.

\section{Related Works}

\subsection{Scanned Compound Image using Digipaper}

There are many works in the literature which focused on scanned compound image compression. The most important works among them namelyDigipaper, H.264/ AVC, JPEG2000 and MMP are discussed in this paper. Digipaper compression algorithm was proposed by D. Huttenocher et al. 1 in which a segmentation method and associated file format to storing images of scanned documents are described. The document is divided into three different layers, containing the background, text, and image. These layers are dissimilar properties, making it attractive to use dissimilar compression techniques to signify the three layers. The compressed background layer is encoded by using Arithmetic encoder; the text layer is using a token-based technique and the image layer is compressed by using augmenting the representation. These representations and the algorithm produced very highly-compressed document files.

The DigiPaper image file design uses token compression and Mixed Raster Content rather than achieving compact representation of scanned document images. One of the troubles with MRC is the segmentation of input images into the background, foreground, and selector layers. While performing this segmentation classification errors occur leading to reduction in image quality.

\subsection{Scanned Compound Image using H.264/ AVC}

Scanned Compound images are mixed with text, graphics and natural pictures. These images are welldefined anisotropic features. The existing image coding principles are ineffective for compressing them. In order to overcome this problem, a compression scheme based on the H.264 intra-frame coding was proposed by Zaghetto et al. ${ }^{9}$ Two fresh intra modes are used in this model to enhance the compression ratio of scanned compound images. They proposed scalar quantization method as the first contribution, where intra-predicted residues are straight quantized and for compression, they used entropy encoded technique. They have also proposed a second technique which is based on colors and index map mode that can be documented as an adaptive vector quantization algorithm. The image blocks were represented by several verities of colors, known as base colors, and index map to compress by using two innovative modes as well as existing intra modes in H.264 are chosen by the Rate-Distortion Optimization (RDO) technique. Their experimental result shows that this method was not only improve the coding competence still more than $10 \mathrm{~dB}$ for scanned compound images. Also parallel performance is provided by H.264/AVC for natural images. The layer-based approach for image compression is mainly used for effective compression .A hard problem in layer-based technique is that accurate segmentation is not achieved in this method, which is greatly affects the quality of the image.

A block-based approach is used for segmentation in H.264 compression. Moreover, Simple characters of 
images such as color values, histogram, mean and gradient are used for classification. Blocks are categorized into dissimilar types, such as text, graphics, and picture image blocks. The experimental results of PSNR and compression ratio of the scanned compound document shown in H.264 provides high image quality and average compression ratio.

\subsection{Scanned Compound Image using JPEG2000}

Optimized JPEG compression was proposed by Tak-Shing Wong et al ${ }^{1}$. In this work, a layer based approach called Mixed Raster Content (MRC) is used for compound document compression. This method includes a multi-layer decomposition representation for scanned compound documents into two image layers called binary mask layer and the foreground layers. The mask layer was used for separate compression techniques. The MRC layered based approach is used by JPEG2000 compression algorithm for image layer and a layer decomposition method corresponding to JPEG2000 is also used for efficient compression

Approximately, all computer-generated, $300 \mathrm{dpi}$ scanned compound documents, still complex, can be represented at complete resolution with high quality at 100:1 compression even though, it is complex for simpler documents, the compression ratio is frequently more than 175:1 KB.With $2 \times 2$ sub-sampling of the image layers; the compression ratio simply exceeds $250: 1$ for the majority documents. For scanned compound documents, the filesize is less by reasonable than $200 \mathrm{~KB}$ down sampling of the image layers. A test set of $300 \mathrm{dpi}$ scanned compound documents yielded a standard compression ratio of 125:1 with $2 \times 2$ layer sub sampling, but the quality was better than other algorithms. The mask serves fine to separate text and other features from the document. Han Oh et

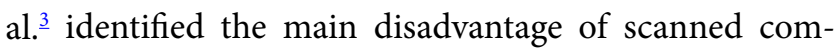
pound document using JPEG2000 since it is based on the assumption that the segmentation process could accurately separate the text and image, but it is not always true. Pixel classification errors may happen while performing segmentation and hence it will affect the overall efficiency of compression scheme.

\subsection{MMP-FP (Flexible Partitioning) and MMP-Text}

Scanned Compound Document Encoding Using Multiscale Recurrent Patterns was proposed by Nelson
$\mathrm{C}$ et $\mathrm{al}^{2 \underline{23}}$. In this work scanned images are divided into smooth and non-smooth blocks and they have segmented the blocks into horizontal or vertical segmentation, finally, the hierarchal tree is formed and selection of the most excellent dictionary block is performed based on the optimization function. They have chosen the code vector which contains minimum cost. Predictive coding is done by using code samples to make predictive blocks. It varies from the original block since it gets a residual block. Later, they have checked the dissimilar predictive modes. The algorithm chosen is applied for dictionary revision and an improvement technique is used for corresponding encoded blocks. MMP uses a scaled version of adaptive pattern matching that contains matching's among the vectors of dissimilar dimensions. The predictive coding technique includes the recognized property of generating residue samples with extremely peaked probability distributions centered on the cost zero that favors the arithmetic coder adaptation process to the basis. The better dictionary adaptation is achived by making use of the predictive fault data error. The next parts of the results were taken from Text blocks of the original image. First, they selected the best dictionary block based on the optimization function. Next they selected the code vector which is having least amount cost. This work assigned a code vector and scale dimension for the least encoded blocks. Finally, the blocks were matched with the dictionary. This procedure is called as pattern matching.

In this paper the all Four Existing schemes namely, JPEG2000, H.264/AVC, DigiPaper and MMP are selected for comparisons. The new scheme proposed in this paper called EMMP is compared with all the four existing techniques and it is observed that the proposed method gives better image quality and high compression ratio for scanned compound images.

\section{Proposed Work}

In the existing works on compression of scanned compound documents, classification schemes were used like Mixed Raster Content (MRC) layer based technique are used. However, they not sufficient for accurately segmenting the text and image blocks. The latest version of scanned compound document classification scheme is block based segmentation. This makes less sensitive to error in block segmentation. In this paper, we have introduce two states of classification; the first state of block classification has been performed with the use of thresholding features like 
gradient vale, color range and color number for dividing the images into text and graphics and the complex blocks. In complex, we applied Rate Distortion Optimization (RDO) segmentation technique to divide again the complex blocks into original text and image blocks. The Main function of this RDO to minimize is $\mathrm{J}(\check{\mathrm{T}})=\mathrm{D}(\breve{\mathrm{T}})+\lambda$ $\mathrm{R}(\breve{\mathrm{T}})$. Here $\mathrm{D}$ and $\mathrm{R}$ are the distortion and rate of each and every block respectively. The cost J of complex blocks in dissimilar modes is calculated and the mode with the minimum $\mathrm{J}$ is selected and the lambda value is constant. Finally, we concatenate the both text blocks and the image blocks which are obtained given from block based and RDO segmentation techniques.

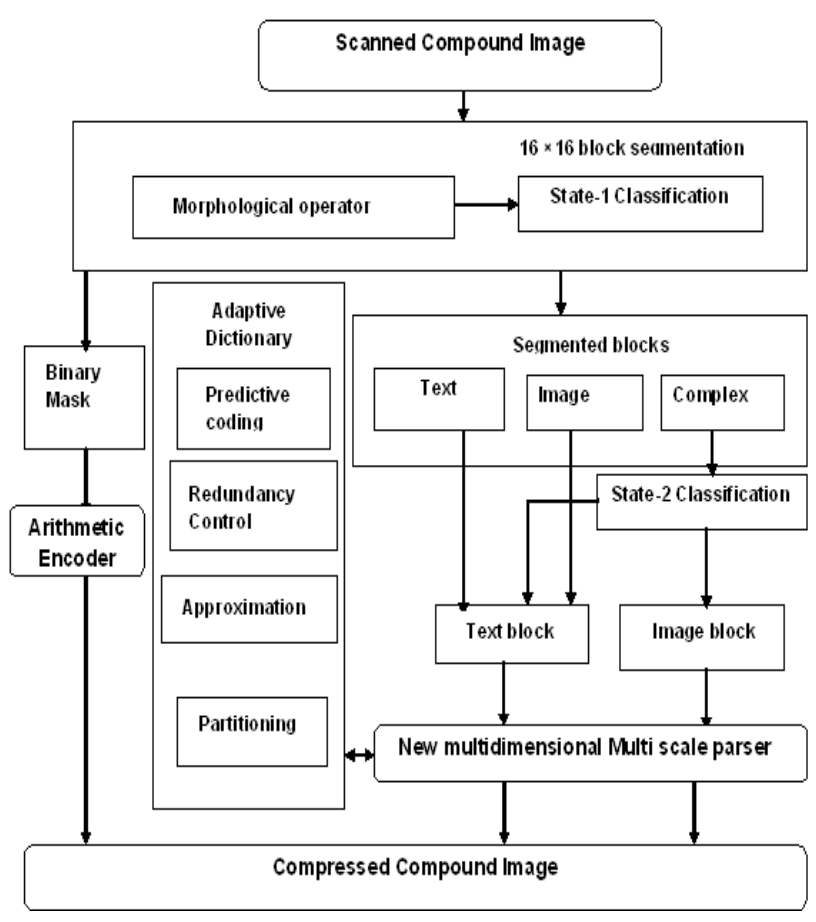

Figure 1. System Architecture.

The existing MMP compression algorithm uses a fixed block partitioning procedure, i.e. vertical or horizontal segmentation. The proposed EMMP applies binary Tree Based segmentation for image and quad tree based Segmentation for the non-smooth image. The text blocks are divided recursively in tree based representation like the left and right child. For example at node V, we applied the binary tree procedure, to separate original vertex into two vertices namely $\mathrm{V}_{1}$ and $\mathrm{V} 2$. Similarly they are further divided into $\mathrm{V} 1_{1}, \mathrm{~V} 1_{2} \mathrm{~V} 2_{1}, \mathrm{~V} 2_{2} \ldots$ recursively. Finally, we have 25 different scales namely $16 \times 16,16 \times 8,16 \times 4 \ldots 1 \times 1$.
Now,the blocks with high costs and the blocks with lower costs are separated in the hierarchal tree order. The blocks with higher costs are pruned away considering only the blocks with low $\operatorname{costs}^{\underline{5}}$ on these blocks, we apply Integer Wavelet Transformations where the original spatial information is converted into frequency signal and that integer values are rounded by the quantization algorithm. Finally, the compressed and encoded texts are obtained. The encoded text blocks are then assigned with the code vectors and scale dimensions for dictionary matching purposes. Adaptive Dictionary for non-smooth side is created for storing away the existing encoded text blocks. The encoded blocks in the dictionary are maintained in the tree format based on rate distortion cost.

Similar to the previous methods the image is segmented into horizontal and vertical segments in this work. Moreover, the proposed EMMP is applied for smooth blocks are subjected to Quad Tree Based Segmentation process where the image blocks are divided recursively into four quadrants continuously ${ }^{6}$. If No further division is possible, then the quad tree will be stopped. From that only the least cost blocks are selected by pruning away the high cost blocks. A similar procedure as in the EMMPText is applied where the low cost blocks selected are subjected to Integer Wavelet Transformations. Here, the image pixels from the spatial domain are converted to the frequency domain. The integer values are rounded off by the use of quantization process and finally the compressed, encoded image blocks are obtained ${ }^{7}$. The adaptive tree based dictionary is created for the smooth part using traditional pattern matching methods. In this adaptive dictionary, we used a predictive coding technique to store the previous encoded sample with the use of arithmetic encoder and also redundancy control mechanism has been proposed to reduce the redundant information. The main advantage of using EMMP encoding technique is that it makes the compression more efficient and the quality of the compressed image is also enhanced. Moreover, the data losses and the complexity are also greatly reduced. The approach used in EMMP compression techniques are summarized in Fig.1.

\subsection{RDO Segmentation}

In the input image, the morphological operators are used to separate the foreground and background layers. R-D stands for Rate Distortion segmentation ${ }^{5}$ where the block segmentation takes place, dividing the compound docu- 
ment into smooth, non-smooth and complex blocks. The smooth blocks are region, containing only the image blocks. Non-smooth blocks are the region, containing only the text/graphics and the complex are the region, containing the intermediate non identification pixel of merged text and image blocks. They are classified based on the threshold, mean and standard deviation of the images and texts ${ }^{8}$. The complex blocks are the blocks intermediate to text blocks and the image blocks. This is again segmented into smooth and non-smooth blocks with the use of RDO algorithm. Distortion is computed using formula shown in equation (1).

$$
D(\tau)=\left\{\begin{array}{l}
5, \text { if } \lambda \leq 15 \\
20, \text { if } 15<\lambda<50 \\
30, \text { otherwise }
\end{array}\right.
$$

where $\lambda$ is the Lagrangian multiplier, $\mathrm{D}(\tau)$ is a Rate distortion of complex blocks; Segmentation technique is used to completely segment the smooth regions from the non-smooth regions easily and quickly ${ }^{9}$. The disadvantage of using other segmentation techniques, during the segmentation process is that the text pixels and the image pixels are overlapped and hence it is not accurate. To avoid such drawback, R-D Segmentation is used where it clearly and accurately classifies the smooth and nonsmooth blocks ${ }^{10}$.

\subsection{Pattern Matching}

The EMMP compression method is used to reduce an enormous selection of data sources. EMMP has lots of vital features that distinguish it from supplementary pattern and dictionary matching algorithms. It uses multiscale approximate pattern matching attached with adaptive dictionary. Conservative pattern matching techniques are moderately accurate for every fixed size image block by using distinct code-vector $\mathrm{V}$ with the same dimensions as B. EMMP uses scale adaptive pattern matching that allows matching among different vector dimensions. If a single vector $B$, of scale $S$ (with dimensions $2^{\llcorner S+S / 2\lrcorner} * 2^{S / 2}$ dictionary of scale with dimensions pixels), is approximated using single vector $\mathrm{V}^{\mathrm{d}}$ of the dictionary of scale $\mathrm{d}$ (with dimensions $2^{\llcorner\mathrm{L}+s / 2\lrcorner} 2^{\mathrm{d} / 2}$ ). This algorithm makes use of a 2-Dimentions scale transformation $\mathrm{T}_{\mathrm{d}}$ and organizes to convert $\mathrm{V}^{\mathrm{d}}$ into a scaled version $\mathrm{V}$ before performing the pattern match. The excellent dictionary block B to symbolize is based on R-D optimization function $\mathrm{J}(\tau)$, specified by

$$
J(\tau)=D(\tau)+\lambda R(\tau)
$$

where $\lambda$ is a Lagrangian multiplier with the intention of weights the relative consequence of the rate distortion $\mathrm{R}(\tau)$ essential for the illustration and the block distortion $\mathrm{D}(\tau)$. Later, on assigning the code-vector that reduce the Lagrangian cost function, the techniques segment the block into two unlike new blocks, namely, $\mathrm{B}_{1}^{\mathrm{S}-1}$ and $\mathrm{B}_{2}^{\mathrm{S}-}$ ${ }^{2}$, each with partially the pixels of the innovative block. The block matching method is recursively used on each sub-block. The total value of the representation cost of the two halves is compared with the cost of in place of $B_{1}$ with a single code-vector which in turn decides whether to segment or not segment the original block. The best possible block partitioning is represented into tree segmentation, which includes all the information desired to produce the ultimate bit stream. The binary tree leaves keep up a correspondence with non-segmented blocks, $\mathrm{B}_{1}$ that are approximated into this one distinctive codevector, acknowledged by its index 'i.' The scale value ' $S$ ' is connected to the block size corresponds to the tree level. While performing decomposition, the compressed image is matched with the dictionary based on code vector and then scaling and $\mathrm{RD}$-cost reverse process are applied ${ }^{11}$.

\subsection{R-D Optimization}

To find optimal or the best solution, we use R-D optimization techniques to evaluate the time and memory requirements of the system. In this work, for lossy type of source coding problems, we use $\mathrm{N}$ coding units of block and $\mathrm{M}$ different quantized choice for each unit. It assured that the rate is $\mathrm{R} \tau$ (i), distortion is $\mathrm{D} \tau$ (i) respectively. For each unit represented as ' $j$, the quantized option is ' $i$ ' is known. Our aim is to find optimal solution $w^{\star}$ that minimizes the total cost distortion given by equation (3).

$$
W^{*}=\arg \operatorname{Min}_{w} \sum_{j=1}^{m} d_{j}(w(j)
$$

Where $W(j)$ is a quantization choice unit for ' $j$.' To find the Lagrangian cost, the Lagrangian multiplier technique is the best choice to solve the optimization problems, The minimization cost is obtained using equation (4).,

$$
J(\tau)=D(\tau)_{j} j(i)+R(\tau)_{j}(i)
$$

From equation (4), an EMMP compression technique is used to find the cost function of quad tree structure and binary tree structure representation of every node. 
Moreover, the objective function is used to minimize the rate and cost distortion of each block. The higher cost blocks are pruned and to keep only lower cost blocks because of the memory reduction and time consumption. We assign scale dimensions of blocks, based on that, we store the encoded blocks into the dictionary.

\subsection{Hierarchical Partitioning}

The essential principle of segmentation lies in the technique is the hierarchical recurrent partitioning. It uses of $16^{*} 16$ block size since it is preferred as block size for effectiveness. The text blocks are split into straight or perpendicularly and these are represented in the dictionary by the numbers $0,1,2,3$, respectively. Two is to be constantly followed by its corresponding dictionary index that contains dissimilar scales of dictionary and they are $16^{\star} 16,16^{\star} 8,16^{\star} 4 \ldots \ldots \ldots .1^{\star} 1$. Two is not desired to be $1^{\star} 1$ sized blocks because it can’t be separated more. Fig.2. shows the choices of partitions obtained by block segmentation.

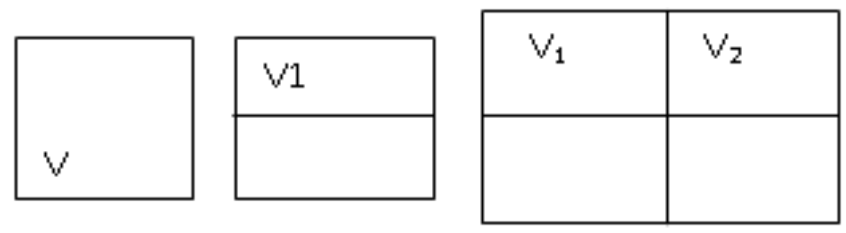

\begin{tabular}{|c|c|c|}
\hline$\vee 1_{1}$ & $\vee 1_{2}$ & \multirow{2}{*}{$\sqrt{ } 2$} \\
\hline$\vee 1_{3}$ & $\vee 1_{4}$ & \\
\hline \multicolumn{2}{|c|}{$\vee 1_{3}$} & $V 1_{4}$ \\
\hline
\end{tabular}

a) Original (b) Binary segmentation (c) quad tree (d) $\mathrm{V}_{1}$ - Node quad tree segmentation

Figure 2. Choices for partitioning.

It is compared with the corresponding part in the original block in order to calculate the distortion. If distortion is a negligible quantity, then that block is taken and coded as the exact partitioning is adopted. If the low amount lies in $\mathrm{V}$ then no partitioning is necessary. If $\mathrm{v} 1$ has least amount then, 0 is added in the dictionary to signify straight partitioning. In addition, $\mathrm{v} 1$ is coded using a dictionary index, whereas $\mathrm{v} 2$ is additional partitioned using same process recursively ${ }^{12}$. Parallel policy is used in case of perpendicular partitioning where the minimum lies in either $\mathrm{v} 3$ or $\mathrm{v} 4$.

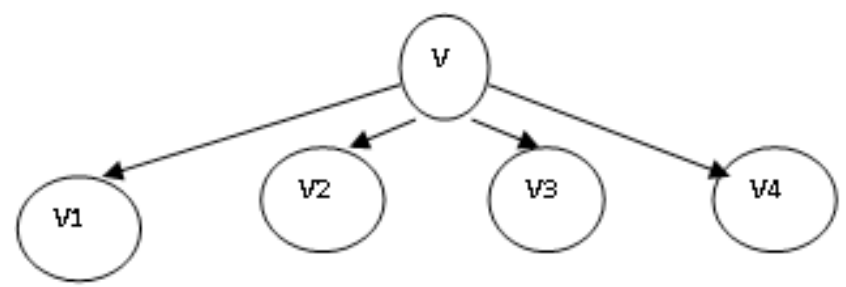

Figure 3. Partitioning of node V as a tree.

In the example shown in Fig 3, $\mathrm{V}$ is partitioned into $\mathrm{v} 1, \mathrm{v} 2, \mathrm{v} 3, \mathrm{v} 4$. In the earlier versions, $\mathrm{v} 1^{13}$ is kept as it is resulting in a binary tree. In our method, $v 1$ is again compared with its equivalent part and distortion is measured. If this distortion is larger than a threshold, it is portioned again. Thus, it becomes a quad tree as each quadrant shown in fig.4.

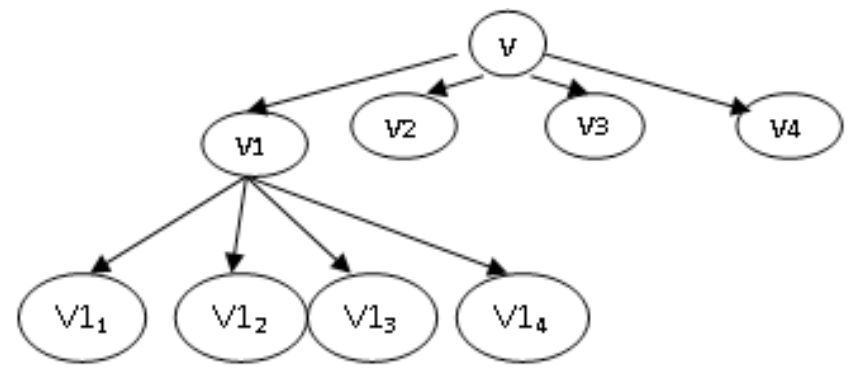

Figure 4. Partitioning of node V as Quad tree.

The dictionary is shaped at both encoding and decoding stages. In earlier versions of MMP used 64 different dictionaries of identical values exclusively distributed from 0 to 255 . This restriction is applied only with those dictionaries whose length is 16 or $8^{14-16}$.

This constraint is made to keep away from the dictionary index, which might reduce the competence of arithmetic encoder. This negative aspect is avoided for dictionaries having length 1 and 2 and 4,8 because it won't overcome the barrier 512 even we permit all the 256 variants. In any case it desires to code all the values below 512.Thus, it will not reduce coding efficiency. The record of dictionaries for which restriction is made are as follows- $16^{\star} 16,16^{\star} 8,8^{\star} 16,8^{\star} 8,4^{\star} 16,4^{\star} 8,2^{\star} 16,2^{\star} 8,1^{\star} 16$, $1^{\star} 8$ ( 64 blocks) and $16^{\star} 4,8^{\star} 4,4^{\star} 4,2^{\star} 4,1^{\star} 4$ (128 blocks) $)^{17}$. The execution time is the most important property of this coding technique. The dictionary learns from innovative patterns that originate from the image and hence 
the dictionary size increases quickly. So, it may need hours to code large images. The results obtained from the experiments are shown in fig. 7 (a), (b) and (c) graphs In addition, the obtained by taking PSNR vs. bpp. PSNR raises quickly when compared to the other methods, after a certain bpp. Consequently the time complexity becomes is exponentially large with the size of the image. It can be reduced up a certain extent by implementing binary search when selecting the best block from the dictionary. The dictionary key is used to search efficiently since, binary searching is considered in this sequence. Linear searching is designed after this range, since it is not at all sequential. This method uses Lagrangian optimization functions. For every five variants, distortions are multiplied by the lambda value. So if lambda is larger than one, greater opportunity for partitioning because it compares distortion via the constant threshold. Consequently, as lambda value increases, the size of compressed file increases with a slight amount of distortion. Lambda $=1$ plays as a value which compromises both size and distortion rates. The distortion procedure calculates standard disparity with respect to a pixel. The block set up in the dictionary is compared with its single block part. Afterwards, their difference is considered for each pixel value. Subsequently the absolute (abs) function is applied followed by a summation (Add) of their value. It is separated by the size of block $(m, n)$ resulted in the standard value ${ }^{\frac{14}{4}}$.

$$
\operatorname{AvgDiff}=\operatorname{Add}(\operatorname{abs}(\text { mat } 1-\operatorname{mat} 2) / m * n
$$

\subsection{Dictionary Formation}

There is an effective dictionary matching technique known as EMMP Adaptive Dictionary matching technique. The adaptive dictionary stores the previously encoded sample blocks based on a different vector (v) and scale (s) and it is followed by hierarchical structure format. The hierarchical data structure used in the adaptive dictionary is tree based data structure. In this structure, a large cost is the root node and the least cost is the child node is calculated. Trees based least blocks are compared with the previously stored encoded blocks in the adaptive dictionary $y^{18}$. If the obtained encoded blocks already exist in the dictionary, it is not stored in the dictionary. If the encoded blocks are not exist, then the blocks are stored in the dictionary. The advantage of using a dictionary is that the transmission time is greatly reduced and there is a high possibility of re-usability.
Dictionary creation is performed using dictionary values shown in the equation(6).

$$
\text { Dict }=\text { QTcode }+ \text { BTcode }(T C F, R D C)
$$

The above equation is newly proposed in this work; where QT and BT are represented by quad tree, binary tree and the Dictionary is created by using of the least cost value of MMP based quad-tree encoded blocks. The binary-tree encoded blocks of the total cost function (TFC), rate and distortion Cost (RDC) for the entire image is calculated.

\subsection{Predictive Coding}

The adaptive dictionary is created using predictive coding techniques, where the existing encoded samples are stored in the dictionary with the use of arithmetic encoder. In that, Prediction is calculated $\mu_{\mathrm{p}}[\mathrm{n}]$ based on earlier samples $[\mathrm{n}]$ is a model from its earlier samples is $\mathrm{B}_{\mathrm{N}+\mathrm{n} .}$ A EMMP uses the adjacent samples of its previously Encoded blocks, And to decode the prediction error signal, $\mathrm{R}_{\mathrm{i}}$ that corresponds to the variation in the middle of the new block, $B_{L}$, and the intra- prediction signal, $P_{i}$. While encoding the image block, EMMP is selected as of accessible prediction models that can achieve the best $\mathrm{RD}$ co-operation. The predictive coding technique used in adaptive dictionary is shown in Fig.5.

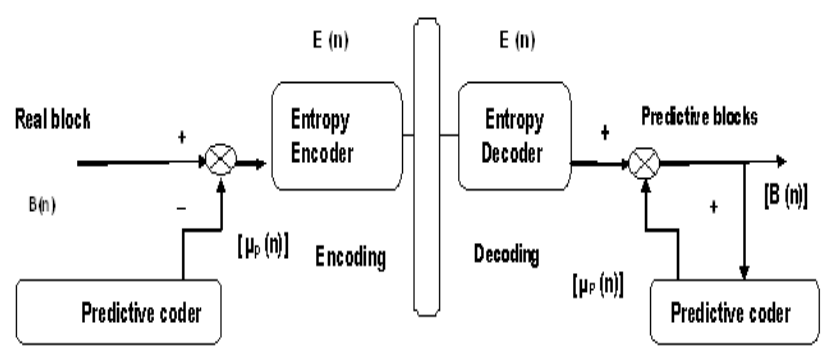

(a)

(b)

Figure 5. Predictive Coding.

Residue block is in use from the original block subtracted into prediction block $\mathrm{R}_{\mathrm{i}}=\mathrm{B}-\mathrm{P}_{\mathrm{i}}$ ). Each image block is transmitted to the decoder side in a predictive form that is encoded via an adaptive arithmetic coder. Encoded values' are indexes to the equivalent residue block after the information is retrieved. The decoder is competent to make a decision the corresponding prediction block, $\mathrm{P}_{\mathrm{i}}$ based on the old encoded adjacent blocks. It decodes the 
hesitancy of the residue block, $\mathrm{R}_{\mathrm{i}}$, and obtains the decoded image block seeing that a result of adding up the decoded residue block with the prediction blocks. $\mathrm{B}(\mathrm{New}$ block $)=$ $\mathrm{R}_{\mathrm{i}}$ (residue block) $+\mathrm{P}_{\mathrm{i}}$ (prediction blocks).

The steps of the proposed predictive coding with EMMP algorithm as follow,

\section{Original image block 'B[n]'}

2. Encoded block $M^{1} \times N^{1}$.

3. Define prediction $\left[\mu_{\mathrm{p}}(\mathrm{n})\right]$ modes to predict block.

4. Each prediction mode $M_{i}$ a. ' $\mathrm{i} \rightarrow$ Determine prediction block $\mathrm{P}_{\mathrm{i}}$ and Residue block $\mathrm{R}_{\mathrm{i}}$

5. Encode E(n) using Arithmetic Encoder

6. Calculate Approximate Residue block $\mathrm{R}_{\mathrm{i}}$

7. Approximation using hierarchal tree $\left(\Gamma \mathrm{M}_{\mathrm{i}}\right)$.
a. Dict $1 \rightarrow$ image $\rightarrow$ BT (TCF, RDC)
b. Dict2 $\rightarrow$ Text $\rightarrow$ QT (TCF, RDC).

8. Fix $\rightarrow$ Decoded image block $=B[n]_{i}$
a. $\mathrm{B}[\mathrm{n}]_{\mathrm{i}}=\mathrm{P}_{\mathrm{i}}+\mathrm{R}_{\mathrm{i}}$
b. Distortion $\mathrm{D}\left(\mathrm{R}_{\mathrm{i}}\right)=\mathrm{d}\left(\mathrm{B}[\mathrm{n}], \mathrm{B}[\mathrm{n}]_{\mathrm{i}}\right)$
c. $\mathrm{RT}\left(\mathrm{R}_{\mathrm{i}}\right) \rightarrow$ Encode the block.

9. Calculate R-D cost.

a. $J M_{i}=D\left(R_{i}\right)+\lambda R T\left(R_{i}\right)+\lambda R_{F}\left(M_{i}\right)$.

b.RT $\rightarrow$ Rate used to encode the Flag for correspond-

ing mode.

10. Fix $M_{i} \rightarrow$ Min $_{\text {RDC }}$ (Rate cost).

11. Encode and transmit $\mathrm{P}_{\mathrm{i}}$ for $\mathrm{M}_{\mathrm{i}}$

12. Encode and transmit $\mathbf{M}_{\mathrm{i}}$; Dictionary indexes of $\Gamma \mathbf{M}_{\mathrm{i}}$.

\section{Experimental Analysis}

Experimental analysis is based on two factors bpp, and PSNR. These are graphs for three dissimilar blocks of images by enhancing bpp on $\mathrm{x}$ axis as well as PSNR on $\mathrm{y}$ axis shown in fig. 7 .

$$
\text { Comp.Ratio }=\text { compsize } * 100 / \text { originalsize }
$$

The PSNR is derived as: It is principally defined by means of the mean- squared - error (mse). It is derived from:

$$
m s e=1 / q \quad \sum_{l=0}^{p-1} \sum_{m=0}^{q-1}\left[I(l, m)-K(l, m]^{2}\right.
$$

In Fig.6 (a) the results of EMMP is matched with the previous version of the algorithms (MMP-Compound) and other techniques. The smooth and non-smooth images scan01, scan02 are compressed by using EMMP and other old encoder techniques.Figure 6 (a) is original scan01.This is Compressed by using our method shown in figure 6 (f). And also we compared with the previous version of the Encoded algorithm such as $\mathrm{DjVu}, \mathrm{H} .264$ / AVC encoded version, JPEG-2000 encoded image and MMP compound encoded. In this paper, a new EMMP method gives the high compression ratio and good PSNR value for the scanned compound document.

Table 1. Experimental results of the image SCAN01 $[\mathrm{dB}]$

\begin{tabular}{|l|l|l|}
\hline Compression Techniques & Rate[bpp] & PSNR [dB] \\
\hline JPEG2000 & 0.30 & 27.29 \\
H.264/AVC & 0.30 & 24.48 \\
DjVu & 0.30 & 23.38 \\
MMP-Compound & 0.30 & 29.98 \\
EMMP & $\mathbf{0 . 3 0}$ & $\mathbf{3 2 . 0 6}$ \\
\hline
\end{tabular}

Table 2. Results from the image SCAN02 [dB]

\begin{tabular}{|l|l|l|l|l|l|}
\hline Rate [bpp] & $\mathbf{0 . 2 0}$ & $\mathbf{0 . 4 0}$ & $\mathbf{0 . 6 0}$ & $\mathbf{0 . 8 0}$ & $\mathbf{1 . 0}$ \\
\hline DjVu & 23.02 & 27.67 & 29.78 & 32.23 & 33.99 \\
\hline JPEG 2000 & 24.89 & 28.34 & 31.42 & 33.65 & 35.72 \\
\hline H.264/AVC & 25.13 & 28.98 & 32.45 & 34.75 & 36.86 \\
\hline $\begin{array}{l}\text { MMP- } \\
\text { Compound }\end{array}$ & 27.86 & 32.57 & 35.65 & 38.32 & 40.41 \\
\hline EMMP & 29.32 & 34.36 & 36.62 & 39.12 & 42.35 \\
\hline
\end{tabular}

The above PSNR and Mean Square error equations are referred from the Gonzales 4th edition book. The meaning of PSNR is similarity apart from that MSE is the summing up over all squared rate differences divided by the image size. The PSNR is reported, after the each channel of that color space. The PSNR value for the lossy type of images and the video compression techniques are 30 and $50 \mathrm{~dB}$, where the top has improved noise ratio. The acceptable values for a wireless transmission range of losses are considered to be $20 \mathrm{~dB}$ to $25 \mathrm{~dB}^{\frac{19}{9}}$. Compression efficiency and computational complexity are computed with the use of matlab. The dictionary optimization functions are used to enhance the EMMP.

Fig.7. It Shows the Rate-distortion plots of the experienced techniques for the scanned test images ${ }^{20}$. Table I highlights the absolute PSNR in $\mathrm{dB}$ value of compression ratios, used for image SCAN01.From these above results one might probably examine the reliability gains achieved by the use of EMMP over each test encoder. Several tests 
OUTLOOK :NEW METERIAL-SPECIAL PROJECTS AVAILABLE

Efficient client services Metal Finishers is one of the sister concern of client

services Electroplaters established in January 2012 with Automatic Zinc (Acid \&

Alkaline) Electroplating process.

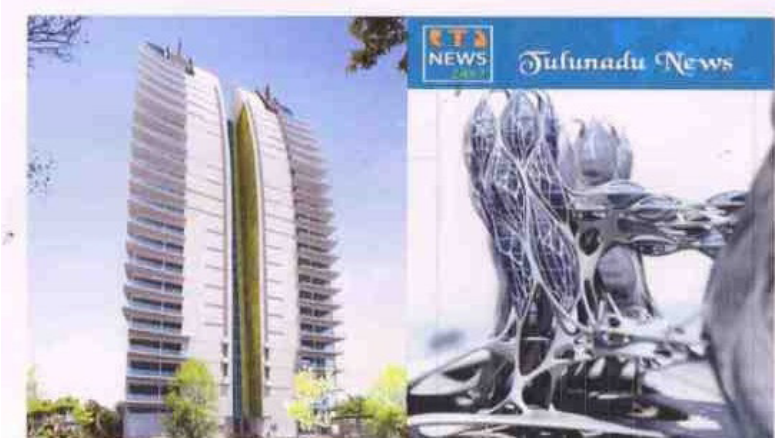

(a) Original Scanned image at 8bpp

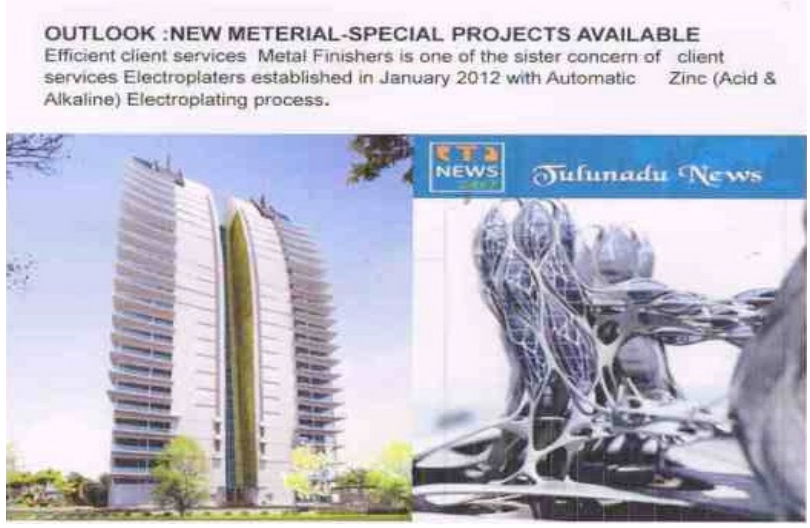

(b)JPEG2000 at $30 \mathrm{bpp}(27.29)$

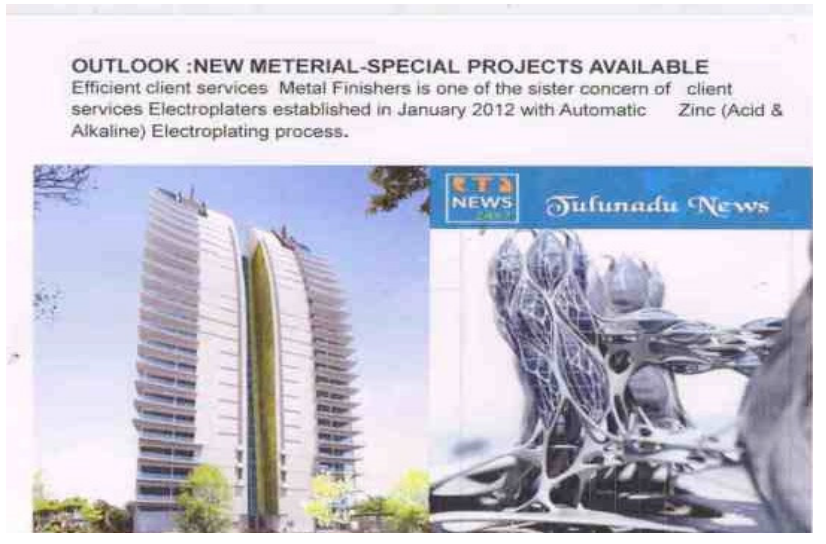

(C) H.264/AVC at $30 \mathrm{bpp}(24.48)$
OUTLOOK :NEW METERIAL-SPECIAL PROJECTS AVAILABLE

Efficient client services Metal Finishers is one of the sister concern of client

services Electroplaters established in January 2012 with Automatic Zine (Acid \&

Alkaline) Electroplating process.

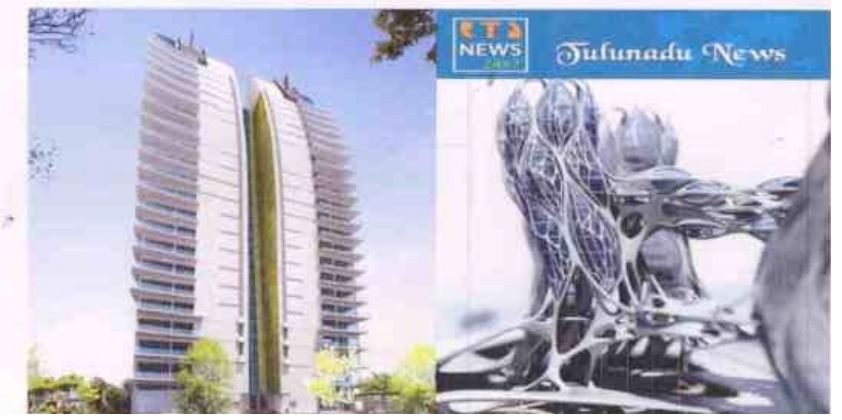

(e) DjVu at $30 \mathrm{bpp}(23.38)$

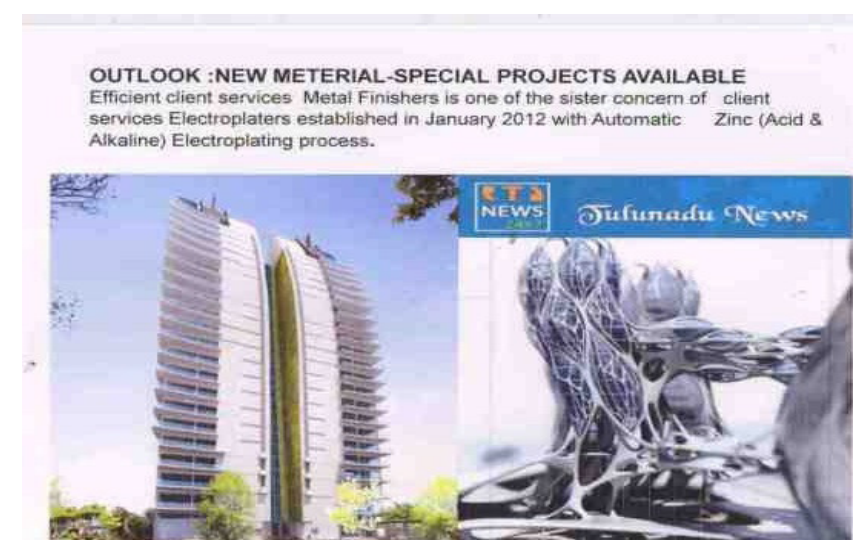

(d) MMP-Compound at $30 \mathrm{bpp}(29.98)$

OUTLOOK :NEW METERIAL-SPECIAL PROJECTS AVAILABLE Efficient clientservices Metal Finishers is one of the sister concern of client services Electroplaters established in January 2012 with Automatic Zinc (Acid \& Alkaline) Electropiating process.

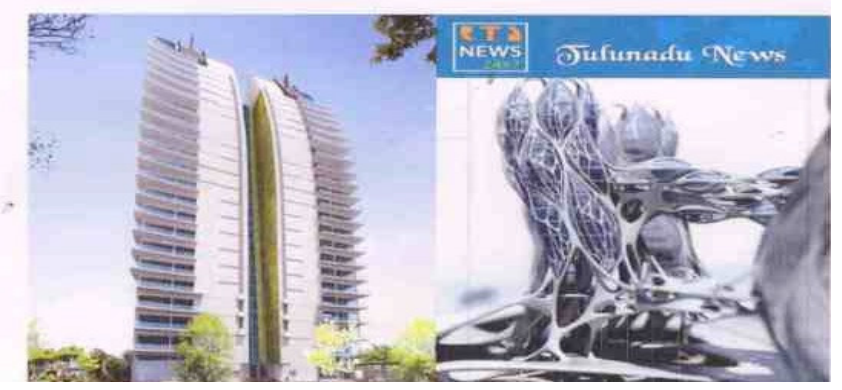

(f) EMMP at $30 \mathrm{bpp}(32.06)$

Figure 6. Various test images of SCAN01 mixed with Text, Image, and Graphics. 
were also performed with the use of Lena images and other scanned compound images and to evaluate the method's robustness of image compression ${ }^{21,22}$. So these test results are demonstrated and giving our methods result is a high performance value ${ }^{23-25}$. Fig.8.shows the results of varies compression techniques applied in scanned compound images.

Text image Result

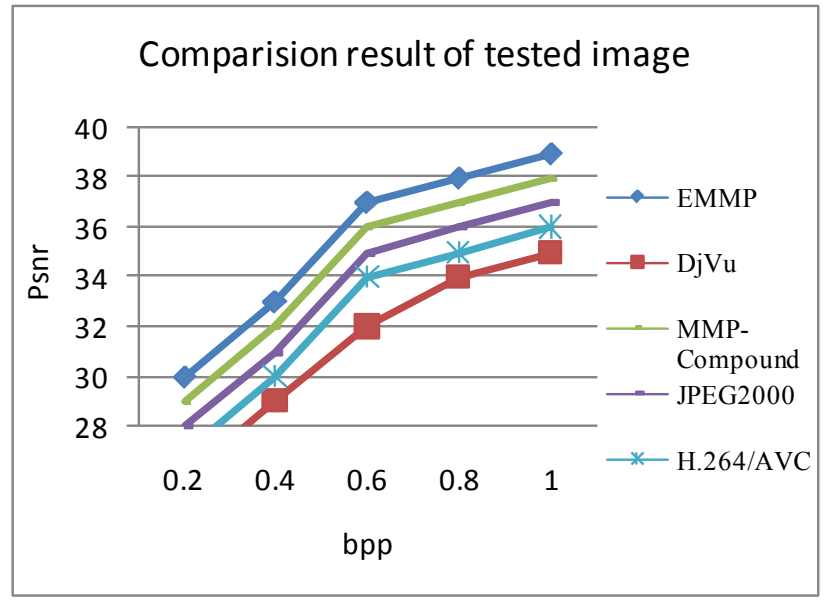

Picture Image Result

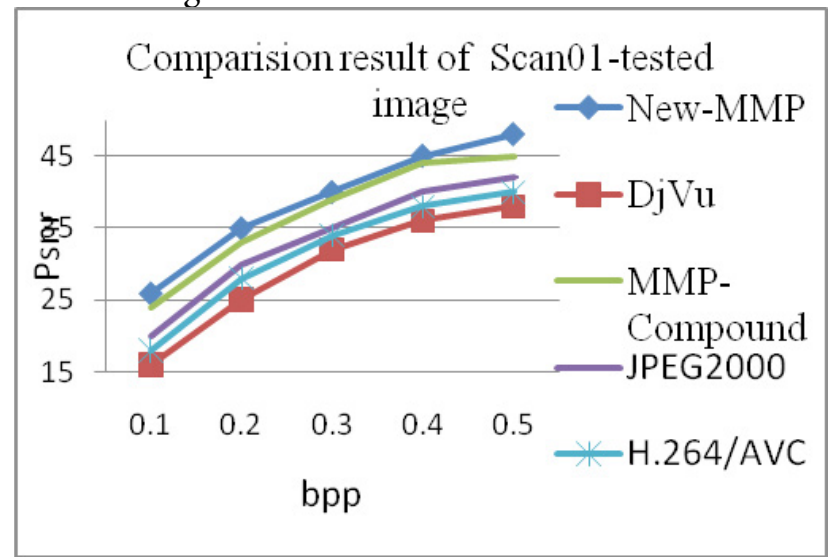

Figure 7. Experimental results for SCAN01 (512×512).
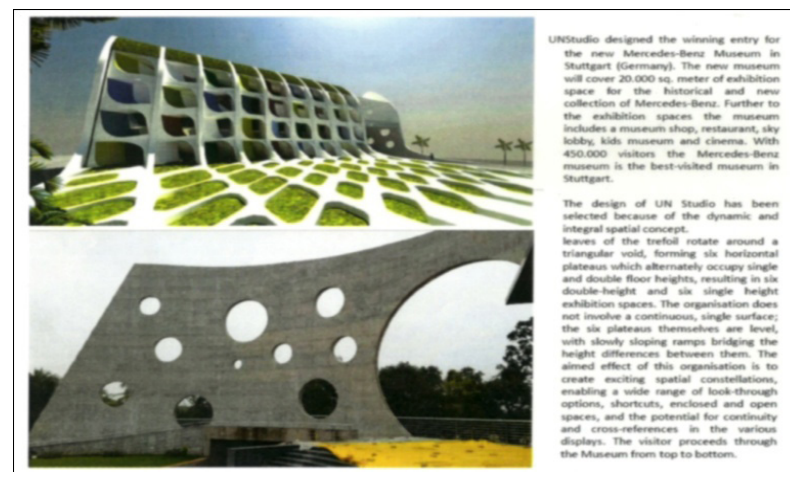

(a)
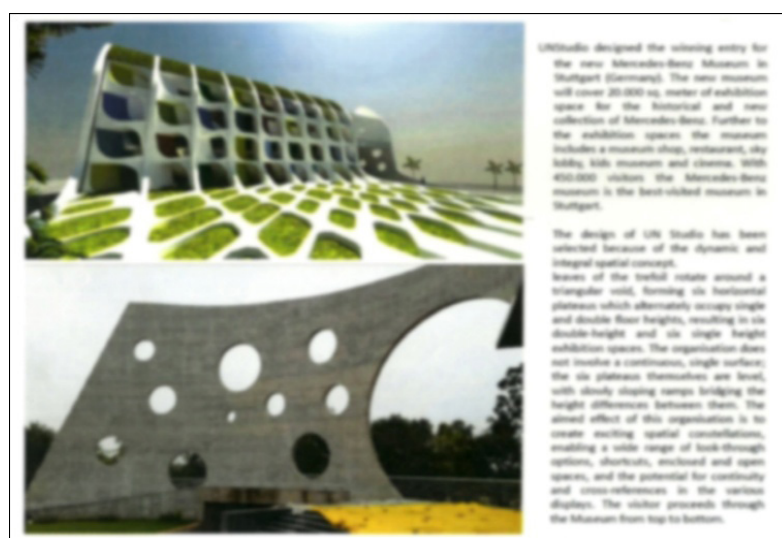

(b)
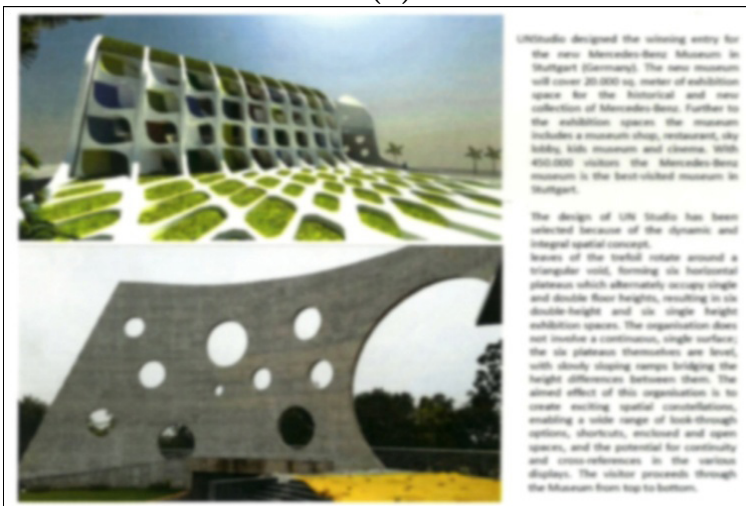

(c)
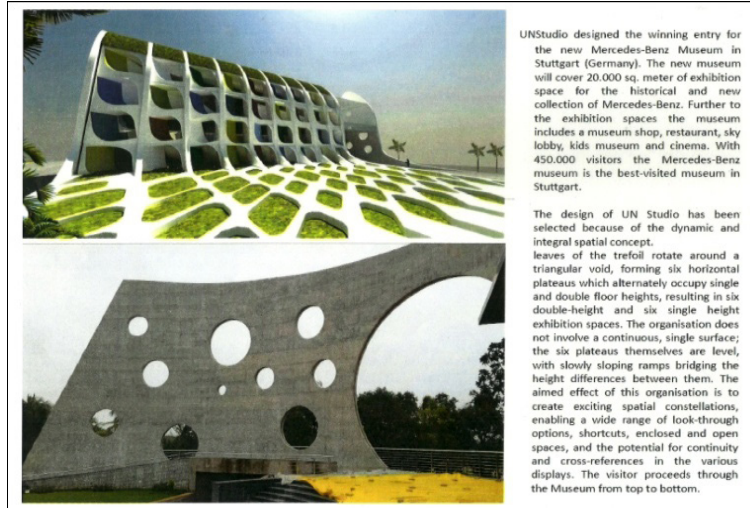

(d)
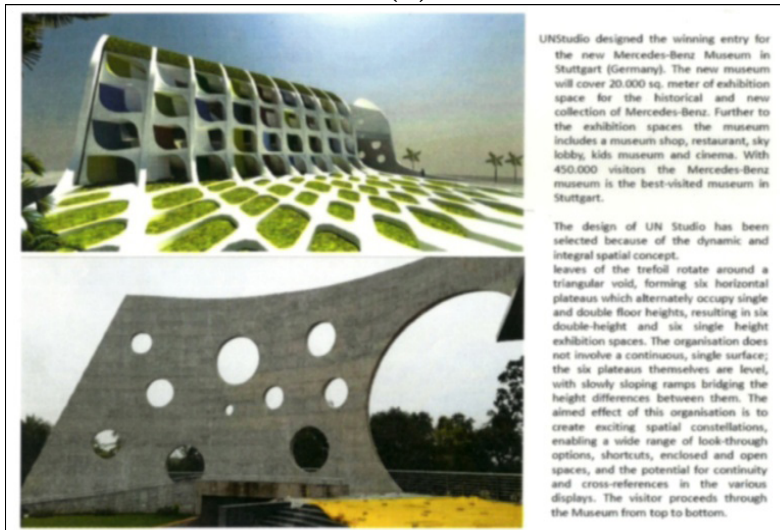

(e) 

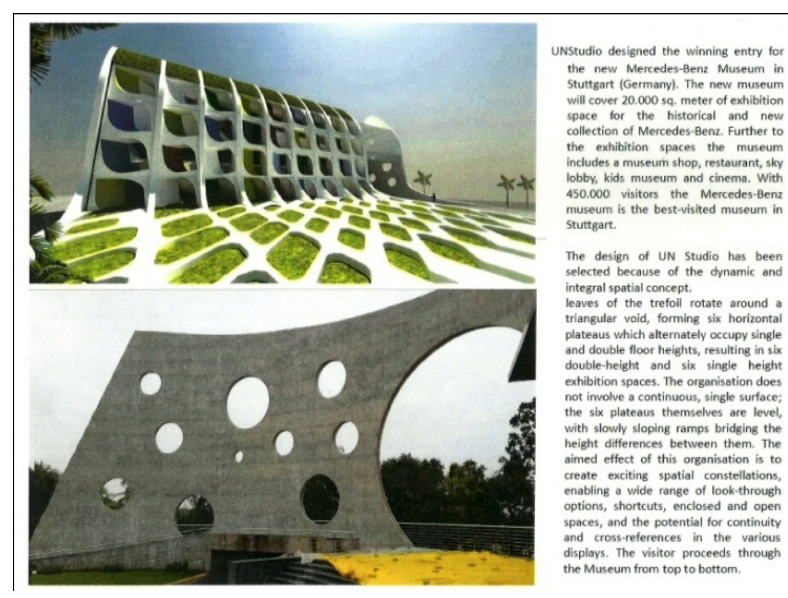

(f)

Figure 8. Details of scanned Compound image (a) scan02. Pgm $(512 \times 512)$ original at $8 \mathrm{bpp}$;(b) DjVu at $0.31 \mathrm{bpp}(25.02$ dB); (c) H.264/AV at $0.30 \mathrm{bpp}$ (26.98 dB); (d) JPEG/2000 at 0.30 bpp (26.25 dB); (e) MMP compound at $0.30 \mathrm{bpp}$ (30.06 dB); (f) EMMP at 0.30bpp (32.49 dB).

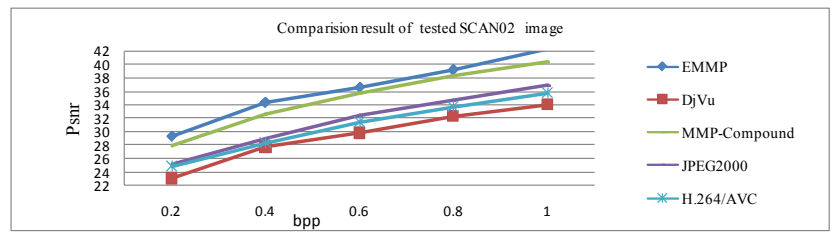

Figure 9. Experimental Graph Result for text and Graphic's Images (SCAN02 $(512 \times 512))$.
Fig. 9 it shows the result of rate-distortion plots of the hardened methods for the used test images ${ }^{26,27}$. Table II highlights the concluding PSNR ratio proposed to the set of compression ratios, for the image Scan02. From these above results one capacity view the constant gains achieved by the use of the EMMP-based approach (Scanned compound) in excess of each tested encoder.

A number of tests were also performed in order to appraise the method's strength next to block miss-classification. These tests confirmed a high performance, yet for regular classification errors ${ }^{28}$.

Table 3 presents three factors value obtained by in built function of Matlab software. The properties are PSNR Value, Compression Ratio and Encoding time. These three values are obtained for Scanned compound images.

Fig. 10. Shows the result of different tested scanned images with same size $(512 \times 512)$. The proposed Algorithm given the Very good PSNR value, high compression ratio and less Encoding Time compared to conventional older algorithms.

\section{Conclusions and Future work}

In this paper a new multi-scale recurring pattern matching Algorithm has been proposed for Scanned compound

Table 3. Results For Varies Tested Images $(512 \times 512)$ Using EMMP

\begin{tabular}{|l|l|l|l|l|}
\hline \multirow{2}{*}{ Image } & \multicolumn{2}{l|}{ PSNR in dB } & Overall Compression \\
\cline { 2 - 4 } & $\mathbf{0 . 3 0}$ bpp & $\mathbf{0 . 6 0}$ bpp & $\mathbf{1 . 0}$ bpp & Ratio (\%) \\
\hline Scanned Compound image 1 & 31.35 & 36.12 & 41.87 & 33.33 \\
\hline Scanned Compound image 2 & 31.05 & 36.01 & 41.56 & 33.52 \\
\hline Scanned Compound image 3 & 32.01 & 36.64 & 42.35 & 33.42 \\
\hline Scanned Compound image 4 & 31.42 & 36.22 & 41.97 & 33.33 \\
\hline Scanned Compound image 5 & 31.30 & 36.11 & 41.80 & 33.51 \\
\hline Scanned Compound image 6 & 30.98 & 35.86 & 40.98 & 33.41 \\
\hline Scanned Compound image 7 & 31.58 & 36.34 & 42.02 & 33.22 \\
\hline Scanned Compound image 8 & 30.96 & 35.78 & 40.97 & 33.52 \\
\hline Scanned Compound image 9 & 32.11 & 36.72 & 42.44 & 33.42 \\
\hline Scanned Compound image 10 & 31.58 & 36.34 & 42.02 & 33.34 \\
\hline Scanned Compound image 11 & 31.03 & 36.08 & 41.40 & 33.52 \\
\hline Scanned Compound image 12 & 31.44 & 36.28 & 41.91 & 33.62 \\
\hline Scanned Compound image 13 & 30.99 & 35.89 & 41.00 & 33.63 \\
\hline Scanned Compound image 14 & 31.24 & 36.10 & 41.82 & 33.61 \\
\hline Scanned Compound image 15 & 32.00 & 36.60 & 42.20 & 33.52 \\
\hline
\end{tabular}


Tested images using EMMP- PSNR Values

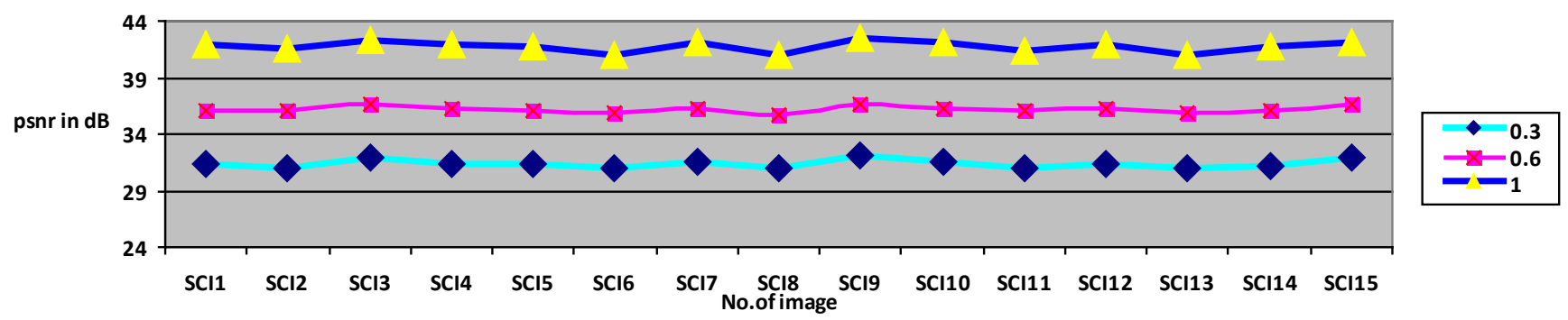

(a)

Tested images using EMMP- Compression ratio

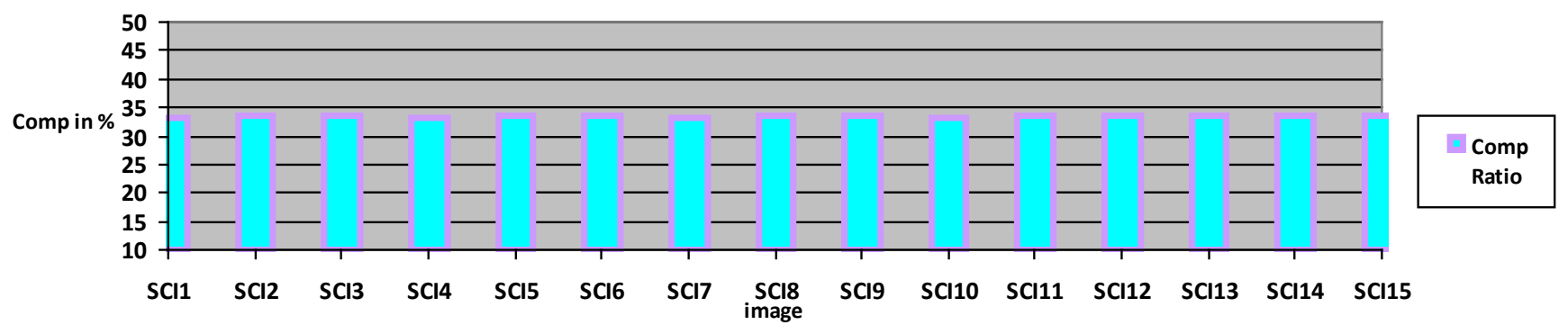

(b)

Figure 10. Experimental Graph Result for Tested scanned Images (512×512), PSNR, Comp Ratio and Encoding Time.

document. This new algorithm utilizes the two stage of block classification scheme, to dividing the image into smooth and non-smooth blocks. Two different EMMPbased encoders (EMMP-image and EMMP-text) are particularly optimized for each image category and also proposed a flexible segmentation scheme like tree based segmentation that is able to utilize the images' arrangement in an additional efficient approach, allowing this technique to enhanced state-of-the-art IWT based encoders for picture images. The optimization applied for the MMP of text/graphics image compression superior its rate-distortion performance for a few images and soft gains over additional basic encoders of up to $8 \mathrm{~dB}$.

This EMMP-scanned compound, produces a compound document encoder by very high-quality ratedistortion and slanted performances. The main criteria utilizing here the universality of EMMP-based encoder's results in a high flexibility to be mistaken block classifications. The experimental results are exposed that it can be used as a most important method over previous ones for scanned document compression. By using this EMMP in scanned images the precision of PSNR value has been improved like $0.30 \mathrm{bpp}(31.35 \mathrm{~dB})$ for our new results. Future works in this trend can be the scheme of a new technique to evaluate the presence of nodules in the segmented scanned images

\section{References}

1. Huttenlocher D, Felzenszwalb P, Rucklidge W. Digipaper: A versatile color document image representation. Proc IEEE Int Conf Image Processing, Kobe, Japan. 1999. p. 219-23. Crossref

2. Wong T-S, Bouman Charles A, Pollak I, Fan Z. A Document Image Model and Estimation Algorithm for Optimized JPEG Decompression. IEEE Transactions on image processing. 2009 Nov; 18(11).

3. Engel D, Stütz T, Uhl A. Assessing JPEG2000 encryption with key dependent wavelet packets. EURASIP Journal on Information Security. 2012; 2012:2. Crossref

4. Oh H, Bilgin A, Marcellin MW. Visually Lossless Encoding for JPEG2000. IEEE Transaction on Image Processing. 2013; 22(1) :189. Crossref PMid:22949058

5. Wari DMH, Ad Enhan D. Layer Based Compound Image Compression. Proceedings of the First Amrita ACM- W 
celebration of Women in Computing (A2CWIC 2010). 2010 Sep 16 \& 17; 209-16. PMid:21053162

6. De Carvalho M, da Silva E, Finamore W. Multidimensional signal compression using multiscale recurrent patterns. Elsevier Signal Processing. 2002 Nov; 82:1559-80. Crossref

7. Filho EBL, da Silva EAB, de Carvalho MB, Pinagé FS. Universal image compression using multiscale recurrent patterns with adaptive probability model. IEEE Trans Image Process. 2008 Apr; 17(4):512-27. Crossref PMid:18390360

8. Rodrigues NMM, de Silva EAB, de Carvalho MB, de Faria SMM, Silva VMM. On dictionary adaptation for recurrent pattern image coding. IEEE Trans Image Process. 2008 Sep; 17(9):1640-53. Crossref PMid: 18701400

9. Said A, Drukarev A. Simplified segmentation for compound image compression. Proceeding of ICIP. 1999; 229-33. Crossref

10. Zaghetto A, de Queiroz RL. Segmentation-driven compound document coding based on H.264/AVC-Intra. IEEE Trans Image Process. 2007 Jul; 16(7):1755-60. Crossref PMid:17605374

11. Yang Q. General Research on Image Segmentation Algorithms. I.J. Image, Graphics and Signal Processing. 2009; 1:1-8. Crossref, Crossref, Crossref, Crossref, Crossref, Crossref, Crossref, Crossref, Crossref, Crossref, Crossref, Crossref

12. Rodrigues NMM, de Silva EAB, de Carvalho MB, de Faria SMM, Silva VMM. On dictionary adaptation for recurrent pattern image coding. IEEE Trans Image Process. 2008 Sep; 17(9):1640-53. Crossref PMid:18701400

13. Smith JR, Chang S-F. Quad-Tree Segmentation for TextureBased Image Query. ACM 2nd International Conference on Multimedia, San Franciso, CA. 1994 Oct. Crossref

14. Chen W-L, Hu Y-C, Liu K-Y, Lo C-C, Wen C-H. VariableRate Quadtree-segmented Block Truncation Coding for Color Image Compression. International Journal of Signal Processing, Image Processing and Pattern Recognition. 2014; 7(1):65-76. Crossref

15. Shusterman E, Feder M. Image Compression via Improved Quadtree Decomposition Algorithms. IEEE Transactions on Image Processing. 1994 Mar; 3(2). Crossref PMid:18291920

16. Kang Y, Yamaguchi K, Naito T, Ninomiya Y. Multiband Image Segmentation and Object Recognition for Understanding Road Scenes. IEEE Transactions on Intelligent Transportation Systems. 2011; 12(4). Crossref

17. Yuanyuan $\mathrm{ZH}$, Zhu Hu. A Hierarchical Segmentation Approach towards Roads and Slopes for Collapse Recognition. International Journal of Signal Processing, Image Processing and Pattern Recognition. 2013; 6(5):15364. Crossref
18. Said A, Pearlman WA. A new fast and efficient image codec based on set partitioning in hierarchical trees. IEEE Trans Circuits Syst. Video Technol. 1996 Jun; 6(3):243-50. Crossref

19. Li Z-W, Ni M-J. Variation Level, Set Method for Multiphase Image Classification. IJ Image, Graphics and Signal Processing. 2011; 5:51-7. Crossref

20. Douda S, Bagri A, El Imrani AA. A reduced domain pool based on DCT for a fast fractal image encoding. Electronic Letters on Computer Vision and Image Analysis. 2011; 10(1):11-23.

21. Sullivan GJ, Wiegand T. Rate-distortion optimization for video compression. Signal Processing Magazine, IEEE. 1998 Nov; 15(6):74-9. Crossref

22. Kim S, Cho N. Lossless compression of color filter array images by hierarchical prediction and context modeling. IEEE Transaction on Circuits and systems for video technology. 2014; $99: 1$. Crossref

23. Moni Sushma Deep K, Srinivasu P. The Importance of feature selection in classification. International Journal on Computer Science and Engineering (IJCSE). 2014 Jan; 6(1). ISSN : 0975-3397.

24. de Carvalho M, da Silva E, Finamore W. Multidimensional signal compression using multiscale recurrent patterns. Signal Process. 2002 Nov; 82:1559-80. Crossref

25. Francisco NC, Rodrigues NMM. Scanned Compound Document Encoding Using Multiscale Recurrent Patterns. IEEE Trans. on Image Processing. 2010 Oct; 19(10). Crossref PMid:20423803

26. de Carvalho M, da Silva E, Finamore W. Multidimensional signal compression using multiscale recurrent patterns. Elsevier Signal Processing. 2002 Nov; 82:1559-80. Crossref

27. Ouni T, Abid M. Scan Methods and Their Application in Image Compression. International Journal of Signal Processing, Image Processing and Pattern Recognition. 2012 Sep; 5(3).

28. Sayadi M, Sakrani S, Fnaiech F, Cheriet M. Gray-level Texture Characterization Based on a New Adaptive Nonlinear Auto-Regressive Filter. Electronic Letters on Computer Vision and Image Analysis. 7(1):40-53,2008.

29. Prabhakar T, Jagan Naveen V, Lakshmi A, Prasanthi, Vijaya Santhi G. Image Compression using DCT and Wavelet Transformations. International Journal of Signal Processing, Image Processing and Pattern Recognition. 2011 Sep; 4(3).

30. Faizal Khan Z, Kannan A. intelligent, approach for Segmenting CT Lung Images using Fuzzy logic with Bitplane. J Electr Eng Technol. 2014; 9(742) 